\title{
Evaluation of enzyme-linked immunosorbent assays and a haemagglutination inhibition tests for the detection of antibodies to Newcastle disease virus in village chickens using a Bayesian approach
}

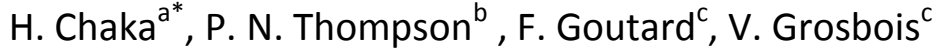

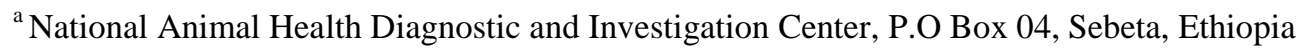 \\ ${ }^{\mathrm{b}}$ Department of Production Animal Studies, Faculty of Veterinary Science, University of Pretoria, Private Bag \\ X04, Onderstepoort 0110, South Africa \\ ${ }^{\mathrm{c}}$ CIRAD, Unite AGIRs, 34398 Montpellier Cedex5, France
}

Corresponding author: email hasscha@yahoo.com Tele: +251 113380894 Fax +251 113380220

\begin{abstract}
Newcastle disease (ND) is an endemic disease in village chickens in Ethiopia with substantial economic importance. The sensitivity $(S e)$ and specificity $(S p)$ of a blocking enzyme-linked immunosorbent assay (bELISA, Svanova Biotech), indirect ELISA (iELISA, Laboratoire Service International) and haemagglutination inhibition (HI) test for ND virus (NDV) antibody detection were evaluated in a Bayesian framework in the absence of a gold standard test, on sera collected from unvaccinated chickens kept under the village production system in household flocks and at markets in two woredas (i.e. districts) of the Eastern Shewa zone, Ethiopia. The outcomes of the iElisa test differed dramatically from those of the two other tests with $92 \%$ of the samples testing positive as compared with less than $15 \%$ for b-Elisa and HI. iElisa results were also inconsistent with previous estimations of Newcastle serological prevalence. The information provided by the iElisa test was thus considered as highly unreliable, probably due to an extremely low specificity, and thus not considered in the
\end{abstract}


Bayesian models aiming at estimating serological prevalence and test performance parameters. Bayesian modelling of $\mathrm{HI}$ and bElisa test results suggested that bELISA had both the highest $S e(86.6 \%$; $95 \%$ posterior credible interval (PCI): $61.8 ; 98.5 \%)$, and the highest Sp (98.3\%; 95\% PCI: 97.2; 99.5\%), while HI had a Se of 80.2\% (95\% PCI: 59.1, 94.3\%), and a $S p$ of $96.1 \%$ (95\% PCI: 95.1; 97.4\%).. Model selection and the range of the posterior distribution of the correlation between bElisa and HI test outcomes for truly seropositive animals (median at 0.461 ; PCI: -0.055 ; 0.894) suggested a tendency for bElisa and HI to detect the same truly positive animals and to fail to detect the same truly positive animals. The use of bELISA in screening and surveillance for NDV antibodies is indicated given its high $S e$ and $S p$, in addition to its ease of automation to handle large numbers of samples compared to HI. The latter can be used as confirmatory test where an ELISA test with moderate or low specificity is used although the likely positive dependence with bElisa implies that $\mathrm{HI}$ and bElisa provide similar information on truly positive animals. Evaluation of commercial ELISA is indicated before their wider use in village chicken populations to avoid erroneous inferences.

Key words: Newcastle disease, diagnostic test evaluation, enzyme-linked immunosorbent assay, haemagglutination inhibition, Bayesian framework, chickens

\section{Introduction}

Newcastle disease (ND), caused by avian paramyxovirus virus type 1 (APMV-1), is a poultry disease with a worldwide economic importance in both commercial settings and village scavenging production systems (Alexander et al., 2004). In Ethiopia, the control of the disease in village chicken flocks, predominant poultry production system, is hampered by low 
veterinary capacity and diagnostic coverage, lack of clear control policies, lack of vaccine suitable for tropical rural conditions (in terms of dosage and thermal stability) and likely also due to low prioritization of development of the sub-sector .

Diagnosis of ND is done by isolation of the virus in embryonated chicken eggs in addition to using serological tests such as various enzyme-linked immunosorbent assay (ELISA) and haemagglutination inhibition (HI) tests (OIE, 2008). During recent decades several polymerase chain reaction (PCR) based assays have been developed for the detection of paramyxovirus nucleic acids in a variety of clinical samples (Gohm et al., 2000; Cattoli and Monne, 2009). While isolation of the virus in embryonated eggs is regarded as a 'gold standard' it takes a relatively long time to culture virus and supply of SPF eggs may also be a problem. The use of molecular diagnostic assays is not within the capability of many laboratories in developing countries because of the equipments, reagents and expertise required. Therefore, serological tests such as ELISA and HI are often relied upon for the laboratory diagnosis of ND or surveys to estimate serological prevalence, indirectly through the detection of antibodies (target condition) produced following infection by ND virus (NDV). However, these tests have limitations due to uncertainty regarding their diagnostic accuracy, as well as, lack of reproducibility under different laboratory conditions (Beard and Wilkes, 1985; Schelling et al., 1999; de Wit et al., 2007). For instance, NDV has been reported to show some degree of cross-reactivity in HI tests with several of the other avian paramyxovirus serotypes, especially APMV-3 psittacine isolates, using polyclonal antisera (Alexander et al., 1983; Lipkind and Shihmanter, 1986; Adair et al., 1989). It was reported that ELISA proved more sensitive and rapid but less economic than HI when used for detection of antibodies against NDV, mostly in vaccinated birds (Adair et al., 1989; Cadman et al., 1997; Tabidi et al., 2004). However, the performance of ELISA and HI tests under 
conditions of field exposure, such as in village chickens, has so far not been well investigated.

The sensitivity $(S e)$ and specificity $(S p)$ of a test are usually estimated by comparison with a reference (gold standard) test that is assumed to reveal unambiguously the true state of animals with respect to disease (Greiner and Gardner, 2000). However, the true state is rarely known because the results of the available diagnostic tests do usually not reflect the true state without uncertainty. The test performance parameters $(S e$ and $S p)$ that define probabilistic relationships between test results and true infection or disease status can be estimated in a Bayesian framework; once these parameters have been estimated, valid inferences on prevalence can be made (Enøe et al., 2000). The Bayesian framework is particularly suited for test performance evaluation, because prior scientific information about the $S e$ and $S p$ of the tests and prior information about the prevalence of the sampled populations can be incorporated (Branscum et al., 2005). The prior distributions could be based on published values from previous studies or on expert opinion (Suess et al., 2002). Basically, distinct tests are assumed to be conditionally independent given the target condition and the theoretical $\mathrm{Se}$ and $S p$ of tests in combination are calculated directly from individual test values. However, for tests that measure similar biologic processes such as serum antibody responses to infectious agents, it is logical to expect that test results will not be independent, conditional on an animal's true status (Gardner et al., 2000). It is important to address such dependence between the $S e$ or $S p$ of pairs of tests because it affects the $S e$ and $S p$ of tests when used in combination (Greiner and Gardner, 2000).

The objective of this study was to evaluate $S e$ and $S p$ two ELISAs and HI tests for the detection of NDV antibodies following infection in chickens under the village poultry production system in Ethiopia using a Bayesian framework. 


\section{Materials and methods}

\section{Serum samples and serological testing}

Chicken sera used in this study were collected during structured, multistage household and markets cross-sectional surveys of village chickens in Eastern Shewa zone, Ethiopia between September 2009 and September 2010 (Chaka et al., 2012; Chaka et al., 2013). Villages and households within villages as well as markets were selected randomly. Households were included provided at least four chickens were present and there was no history of vaccination. From each selected household flock, only chickens over 8 weeks old were sampled. The chickens were kept in a scavenging village production system. A total of 1899 individual chicken sera which constituted the "household dataset" were collected during two seasons of the year ( $n=1018$ for wet season and $n=881$ for dry season) from villages located in 4 kebeles (i.e. communes) of 2 woredas (i.e. Districts), and tested with a blocking ELISA (bELISA) and HI tests. Sera from markets, constituting the "market dataset" were obtained during two different seasons ( $n=204$ for wet season and $n=233$ for dry season) in 4 markets of the same 2 woredas where household samples have been collected and tested with the bELISA, an indirect ELISA (iELISA) and HI tests. Broadly, the wet season in the study area corresponds to the months of June to September, some- times extending to mid-October, while the dry season lasts from October to May. None of the study households vaccinated their chickens against ND. The target population was the village chickens in the two woredas of Eastern Shewa Zone of Oromia regional state, Ethiopia. It was considered that serological prevalence could vary among seasons (wet vs. dry), surveys (household vs. market) and woredas (Ada'a vs. Adami Tullu and Jido Kombolcha (hereafter ATJK).

Blood samples were collected from the brachial vein in 3-mL disposable syringes using a new syringe and needle for each chicken, allowed to clot and were kept at room temperature 
for serum collection. Serum was collected in $2-\mathrm{mL}$ cryovial tubes and kept at $-20^{\circ} \mathrm{C}$ until testing. The analysis of the serum was performed at the National Animal Health Diagnostic and Investigation Center (NAHDIC), Ethiopia using two commercial ELISAs namely: blocking ELISA (bELISA), indirect ELISA (IELISA) and Haemagglutination inhibition test, indicated below, following either the manufacturer's or OIE recommendations. Other details pertinent to the test principles' and interpretation of the results is provided as a supplementary martial.

Blocking ELISA

Blocking ELISA was performed using Svanovir ${ }^{\mathrm{TM}}$ NDV-Ab test kits (Svanova Biotech, Uppsala, Sweden) using the procedure described by manufacturer and validated by Czifra $e t$ al. (1996). The test was developed to detect specific serum antibodies against a wellconserved epitope present in APMV-1 strains.

\section{Indirect ELISA}

The LSIVET AVI NDV ${ }^{\circledR}$ ELISA was obtained from Laboratoire Service International (LSI, Lissieu, France) and is based on the principle of indirect ELISA (iELISA) and designed to be used on individual poultry serum. The iELISA works on the principle of recognition of antiNDV antibodies, attached to a plate coated with viral antigen (nucleoprotein), by antibodies produced in another species against chicken antibodies.

\section{Haemagglutination inhibition test}

The HI test is based on the principle that the haemagglutinin on the viral envelope can bring about the agglutination of chicken red blood cells (RBC) and that this can be inhibited by specific antibodies (Alexander et al., 2004). The test was performed as described by the World organization for Animal Health (OIE, 2008). 
Prior information for the test parameters ( $S e$ and $S p$ ) were obtained from published papers (Czifra et al., 1996; Koch et al., 1998; Gohm et al., 1999; Hauslaigner et al., 2009) for the bELISA and HI test, and manufacturers' claim (Laura Fournier, 2011, personal communication for iELISA) while information on the prevalence was obtained from available literature (Zeleke et al., 2005b; Regassa et al., 2007; Getachew, 2009) and beta prior distributions were elicited using beta buster software (www.epi.ucdavis.edu/diagnostictest/). Prior distribution of the $S e$ and $S p$ of the three tests and the population prevalence were modelled as beta $(\alpha, \beta)$ distributions where parameters $\alpha$ and $\beta$ were derived from the most likely value (mode) and the $5^{\text {th }}$ or $95^{\text {th }}$ percentile of the $S e, S p$ and prevalence (Table 1). Because the ELISAs and HI are all detected NDV antibodies, we regarded these tests as possibly conditionally dependent. We assumed constant test performances irrespective of season or survey type so that test accuracy estimates were interpreted as estimates of the average $S e$ or $S p$ across populations (Branscum et al., 2005).

Table 1 Available information on test sensitivity (Se) and specificity (Sp) and prevalence (pi), and corresponding prior distributions.

\begin{tabular}{|c|c|c|c|c|c|c|c|}
\hline \multirow[t]{2}{*}{ Parameter } & \multicolumn{3}{|c|}{ Prior knowledge } & \multicolumn{4}{|c|}{ Prior distribution } \\
\hline & Mode & $\begin{array}{l}5^{\text {th }} \\
\text { centile }\end{array}$ & $\begin{array}{l}95^{\text {th }} \\
\text { centile }\end{array}$ & alpha & beta & Mean & $95 \%$ PI \\
\hline Se bELISA & 0.95 & 0.70 & & 10.8 & 1.5 & 0.88 & $0.65 ; 0.99$ \\
\hline $\mathrm{Se} \mathrm{HI}$ & 0.80 & 0.50 & & 7.55 & 2.64 & 0.74 & $0.45 ; 0.95$ \\
\hline$S p$ bELISA & 0.95 & 0.70 & & 10.78 & 1.51 & 0.88 & $0.65 ; 0.99$ \\
\hline Sp HI & 0.95 & 0.70 & & 10.78 & 1.51 & 0.88 & $0.65 ; 0.99$ \\
\hline$p i$ & 0.10 & & 0.50 & 1.49 & 5.38 & 0.216 & $0.02 ; 0.56$ \\
\hline rho & \multicolumn{3}{|c|}{ no prior knowledge } & \multicolumn{2}{|c|}{ uniform $(-1 ; 1)$} & 0 & $-0.90 ; 0.90$ \\
\hline
\end{tabular}


The $S e$ and $S p$ of the bELISA and HI test and the seroprevalences were estimated in a Bayesian framework using the model described in Branscum et al., (2005) modified to fit the specific sampling design of our study. In this modified version, the $S e$ and $S p$ parameters for bELISA and HI were estimated using both data from households and markets. Since the tests measured broadly the same biological process (antibodies following NDV infection), models with conditional dependencies were considered. Moreover, alternative models as regard with variation in serological prevalence were fitted to the data. The models were fitted using the freeware programme WinBUGS version 1.4 (Spiegelhalter et al., 2003). The program uses Markov Chain Monte Carlo (MCMC) sampling to obtain a Monte Carlo (MC) sample from the posterior distribution. For each model considered, three chains of one hundred and twenty thousand samples were produced. The initial 20000 samples of each chain were discarded as burn-in. To avoid auto-correlation of successive samples, the remaining 100000 samples of each chain were thinned by selecting every 5th sample of the chain. Posterior distributions were thus derived from three chain of 20000 uncorrelated samples. Convergence of the MCMC chains was assessed by visual inspection of Gelman-Rubin diagnostic plots (Toft et al., 2007). Additionally, unimodality of the posterior distributions and good chain mixing were checked by examining density and trace plots, respectively (Toft et al., 2007) The deviance information criterion (DIC) was used to compare models, with a smaller value indicating better fit. The posterior mean and 95\% posterior credible intervals (PCI, 2.5 and 97.5 percentiles), for each of the parameter estimates ( $S e, S p$, and true prevalences) were presented.

The parameters (rho) reflecting the correlation of the tests' outcomes conditionally on the true serological status of the animals were also included in some of the candidate models; rho $\mathrm{D}$ and $r h o \mathrm{Dc}$ represent the correlation between the tests' outcomes among truly seropositive animals and truly seronegative animals, respectively. Since we evaluated 
serological tests, the focal epidemiological status was the presence or absence of antibodies to NDV. DIC based model selection and examination of posterior distributions were considered to assess conditional dependence (Branscum et al., 2005).

\section{Sensitivity analysis}

Sensitivity of posterior distributions to prior distributions was assessed by running the Bayesian analysis with non-informative priors for all or part of the model's parameters. Overlapping of PCI was considered as evidence of model robustness.

\section{Results}

\section{Descriptive results}

Cross classification of the serological test results is shown in Table 2. A striking feature of the results in the table is for the sample results collected in markets and tested with the three serological tests, that showed extremely high proportion of positive samples according to the iElisa test (403 positives out of 437 samples tested with iElisa) as compared to the proportion of positive samples according to HI (56 positives out of 437 samples) or bElisa ( 27 positives out of 437 samples). The information provided by the iElisa test was thus considered as highly unreliable. iElisa results were thus not considered in the Bayesian models aiming at estimating serological prevalence and test performance parameters. 
Table 2 Cross classified serological test results from chicken serum samples from households and markets in Eastern Shewa zone, Ethiopia between September 2009 and September 2010.

\begin{tabular}{|c|c|c|c|c|c|c|c|c|c|c|c|}
\hline \multirow{3}{*}{$\begin{array}{l}\text { Data } \\
\text { set }\end{array}$} & \multirow[t]{3}{*}{$\mathrm{n}^{*}$} & \multicolumn{2}{|c|}{ Serological tests } & \multicolumn{8}{|c|}{ Combination of test results } \\
\hline & & \multirow[b]{2}{*}{ Test1 } & \multirow[b]{2}{*}{ Test2 } & \multicolumn{4}{|c|}{ (Wet season) } & \multicolumn{4}{|c|}{ (Dry season) } \\
\hline & & & & ++ & +- & -+ & -- & ++ & +- & -+ & -- \\
\hline $1^{a}$ & 1899 & bELISA & $\mathrm{HI}$ & 41 & 15 & 37 & 925 & 73 & 23 & 10 & 775 \\
\hline $2^{b}$ & 437 & iELISA & bELISA & 13 & 173 & 0 & 18 & 14 & 203 & 0 & 16 \\
\hline $2^{b}$ & 437 & iELISA & $\mathrm{HI}$ & 19 & 167 & 2 & 16 & 32 & 185 & 3 & 13 \\
\hline $2^{b}$ & 437 & bELISA & HI & 7 & 6 & 14 & 177 & 7 & 7 & 28 & 191 \\
\hline
\end{tabular}

\section{Evaluation of test performance}

Twenty eight alternative models, differing with regard to prevalence variation patterns and conditional dependencies between test results were fitted to bElisa and HI test results (Table 3). Gelman-Rubin diagnostic statistics that did not converge towards 1, bimodal posterior distribution and poor chain mixing for at least one parameter revealed poor model convergence for eight of these 28 models. The 8 models which failed to converge all included a parameter reflecting conditional dependence between $\mathrm{HI}$ and bElisa test results for truly seronegative animals. Among the models showing good convergence, the lowest DIC model included a parameter reflecting conditional dependence between $\mathrm{HI}$ and bElisa test results for truly seropositive animals and parameters reflecting variation in seroprevalence according to seasons, Woredas and surveys. The second lowest DIC model was similar except that seroprevalence varied according to seasons and Woredas but not surveys Although the former model had a lower DIC, it included 8 parameters to account for quite limited variation in 
Table 3 Model selection

\begin{tabular}{|c|c|c|}
\hline Prevalence variation & Conditional dependencies & DIC \\
\hline \multirow[t]{4}{*}{$\begin{array}{l}\text { Weradas, seasons and surveys ( } 8 \\
\text { populations) }\end{array}$} & $\begin{array}{l}\text { For both true negatives and true } \\
\text { positives }\end{array}$ & Poor convergence \\
\hline & For true positives only & 182.98 \\
\hline & For true negatives only & Poor convergence \\
\hline & Conditional independence & 188.914 \\
\hline \multirow[t]{4}{*}{$\begin{array}{l}\text { Weradas and seasons ( } 4 \\
\text { populations) }\end{array}$} & $\begin{array}{l}\text { For both true negatives and true } \\
\text { positives }\end{array}$ & 187.78 \\
\hline & For true positives only & 186.81 \\
\hline & For true negatives only & 189.2 \\
\hline & Conditional independence & 188.17 \\
\hline \multirow[t]{4}{*}{$\begin{array}{l}\text { Seasons and surveys ( } 4 \\
\text { populations) }\end{array}$} & $\begin{array}{l}\text { For both true negatives and true } \\
\text { positives }\end{array}$ & Poor convergence \\
\hline & For true positives only & 192.72 \\
\hline & For true negatives only & Poor convergence \\
\hline & Conditional independence & 195.85 \\
\hline \multirow[t]{4}{*}{$\begin{array}{l}\text { Weradas and surveys ( } 4 \\
\text { populations) }\end{array}$} & $\begin{array}{l}\text { For both true negatives and true } \\
\text { positives }\end{array}$ & Poor convergence \\
\hline & For true positives only & 195.88 \\
\hline & For true negatives only & Poor convergence \\
\hline & Conditional independence & 198.29 \\
\hline \multirow[t]{4}{*}{ Weradas (2 populations) } & $\begin{array}{l}\text { For both true negatives and true } \\
\text { positives }\end{array}$ & 198.63 \\
\hline & For true positives only & 197.7 \\
\hline & For true negatives only & 200.16 \\
\hline & Conditional independence & 199.11 \\
\hline \multirow[t]{4}{*}{ Seasons (2 populations) } & $\begin{array}{l}\text { For both true negatives and true } \\
\text { positives }\end{array}$ & 197.75 \\
\hline & For true positives only & 197.55 \\
\hline & For true negatives only & 198.21 \\
\hline & Conditional independence & 197.91 \\
\hline \multirow[t]{4}{*}{ Surveys (2 populations) } & $\begin{array}{l}\text { For both true negatives and true } \\
\text { positives }\end{array}$ & Poor convergence \\
\hline & For true positives only & 193.72 \\
\hline & For true negatives only & Poor convergence \\
\hline & Conditional independence & 195.85 \\
\hline
\end{tabular}

seroprevalence (See Table 4) and it was less robust than the later model (see sensitivity analysis below). The later model, which was much more robust to changes in prior distributions (see sensitivity analysis below) and included only 4 parameters to account for seroprevalence variation was thus preferred. In this model, the $S e$ of bELISA and HI was estimated at $86.6 \%$ (95\% PCI: 61.8; 98.5\%), and 80.2\% (95\% PCI: 59.1; 94.3\%), respectively (Table 3). Specificity estimates were high for bELISA and HI: 98.3\% (95\% PCI: 97.2; 99.5\%) and 96.1\% (95\% PCI: 95.1; 97.4\%), respectively. Posterior estimates for NDV seroprevalence in the Ada'a woreda were 6.3\% (95\% PCI: 4.1; 9.7\%) during the wet season 
and $13.3 \%$ (95\% PCI: 9.7; 19.1\%) during the dry season. Posterior estimates for NDV seroprevalence were lower in the ATJK woreda and varied less between seasons: $4.3 \%$ (95\% PCI: $2.5 ; 7.2 \%)$ during the wet season and $6.2 \%$ (95\% PCI: $3.8 ; 9.9 \%)$ during the dry season. In general the parameter posterior estimates were quite close to prior distribution mean.

Table 4 The posterior mean and 95\% posterior credibility interval (PCI), under different model specifications, for the sensitivity and specificity of the bELISA and HI tests, the population prevalence ( $p i)$ and the correlation coefficient $(r h o D)$ of bElisa and $\mathrm{HI}$ test results for true positive animals.

\begin{tabular}{|c|c|c|c|c|}
\hline \multirow[t]{2}{*}{ Parameter } & \multicolumn{2}{|c|}{ Model $1^{\mathrm{a}}$} & \multicolumn{2}{|c|}{ Model $2^{b}$} \\
\hline & median & $95 \% \mathrm{PCI}$ & median & $95 \%$ PCI \\
\hline Se bELISA & 0.866 & $0.618 ; 0.985$ & 0.803 & $0.453 ; 0.983$ \\
\hline$S p$ bELISA & 0.983 & $0.972 ; 0.995$ & 0.983 & $0.972 ; 0.995$ \\
\hline $\mathrm{Se} \mathrm{Hi}$ & 0.802 & $0.591 ; 0.943$ & 0.729 & $0.419 ; 0.913$ \\
\hline Sp HI & 0.961 & $0.951 ; 0.974$ & 0.960 & $0.950 ; 0.971$ \\
\hline pi Ada'a, wet season, markets & & & 0.091 & $0.036 ; 0.195$ \\
\hline pi Ada'a, wet season, households & 0.063 & $0.041 ; 0.097$ & 0.067 & $0.041 ; 0.125$ \\
\hline pi Ada'a, dry season, markets & & & 0.081 & $0.032 ; 0.181$ \\
\hline pi Ada'a, dry season, households & 0.132 & $0.097 ; 0.191$ & 0.159 & $0.111 ; 0.288$ \\
\hline pi ATJK, wet season, markets & & & 0.043 & $0.011 ; 0.117$ \\
\hline pi ATJK, wet season, households & 0.043 & $0.025 ; 0.072$ & 0.049 & $0.028 ; 0.095$ \\
\hline pi ATJK, dry season, markets & 0.062 & $0.038 ; 0.099$ & 0.043 & $0.010 ; 0.126$ \\
\hline pi ATJK, dry season, households & & & 0.077 & $0.046 ; 0.146$ \\
\hline rhoD & 0.461 & $-0.055 ; 0.894$ & 0.664 & $0.002 ; 0.947$ \\
\hline
\end{tabular}


In the two lowest DIC models, the posterior distribution of the correlation between bElisa and HI test results for true positive animals was wide but shifted towards positive values (for the selected model: median at 0.461 ; PCI: $-0.055 ; 0.894)$. This suggested a tendency for bElisa and $\mathrm{HI}$ to detect the same truly positive animals and to fail to detect the same truly positive animals. However, the wide posterior distribution of this parameter reflected high uncertainty on the strength of the dependency between test results.

\section{Sensitivity analysis}

An analysis of the sensitivity of posterior distribution to prior distributions was performed for the selected model (i.e. the model where prevalence varies between seasons and woredas and which includes a parameter for dependence of test results for truly positive animals Table 3). The results of this sensitivity analysis, presented in Table 5, reveal that consistent posterior distributions are obtained whenever an informative prior is used for the sensitivity of the bElisa test. Indeed, a model where non informative priors are used for all the parameters except for the sensitivity of bElisa produces posterior distributions which are very similar to those obtained from a model where informative priors are used for all the parameters. In addition, the informative prior used for the sensitivity of bElisa does not seem overly informative: it is a beta distribution in which sensitivity is allowed to range between 0.65 and 0.99. By contrast sensitivity analysis for the lowest DIC model (i.e. the model where prevalence varies between seasons, woredas and surveys and which includes a parameter for dependence of test results for truly positive animals; Table 3) indicated that this model lacked robustness. As shown in Table 6, using non informative priors for some of this model's parameters resulted in poorly converging models that produced inconsistent posterior parameter distributions. This is one of the reasons why this model was eventually not selected. 
Table 5 Sensitivity analysis: posterior estimates for the sensitivity and specificity of bELISA and iELISA, and prevalence, in a four population Bayesian model including conditional dependence between test results for truly positive animals.

\begin{tabular}{|c|c|c|c|c|c|c|c|}
\hline Parameter & Informative priors & $\begin{array}{c}\text { Non informative for } \\
\text { prevalences }\end{array}$ & $\begin{array}{c}\text { Non informative } \\
\text { for test } \\
\text { performance } \\
\text { parameters }\end{array}$ & $\begin{array}{c}\text { Non informative } \\
\text { priors for all } \\
\text { parameters except } \\
\text { sensitivities }\end{array}$ & $\begin{array}{c}\text { Non informative } \\
\text { priors for all } \\
\text { parameters except } \\
\text { sensitivity bElisa }\end{array}$ & $\begin{array}{c}\text { Non informative } \\
\text { priors for all } \\
\text { parameters except } \\
\text { sensitivity HI }\end{array}$ & $\begin{array}{c}\text { Non informative } \\
\text { priors for all } \\
\text { parameters }\end{array}$ \\
\hline & median $[95 \% \mathrm{PCI}]$ & median $[95 \% \mathrm{PCI}]$ & median $[95 \% \mathrm{PCI}]$ & median $[95 \% \mathrm{PCI}]$ & median $[95 \% \mathrm{PCI}]$ & median $[95 \% \mathrm{PCI}]$ & median $[95 \% \mathrm{PCI}]$ \\
\hline Se bELISA & $0.866[0.618 ; 0.985]$ & $0.869[0.628 ; 0.986]$ & $0.524[0.211 ; 0.969]$ & $0.872[0.628 ; 0.987]$ & $0.889[0.647 ; 0.988]$ & $0.527[0.148 ; 0.956]$ & $0.195[0.11 ; 0.902]$ \\
\hline$S p$ bELISA & $0.983[0.972 ; 0.995]$ & $0.982[0.972 ; 0.995]$ & $0.980[0.969 ; 0.995]$ & $0.983[0.972 ; 0.997]$ & $0.981[0.971 ; 0.996]$ & $0.981[0.968 ; 0.996]$ & $0.979[0.963 ; 0.995]$ \\
\hline $\mathrm{Se} \mathrm{Hi}$ & $0.802[0.591 ; 0.943]$ & $0.807[0.599 ; 0.944]$ & $0.526[0.214 ; 0.963]$ & $0.799[0.594 ; 0.942]$ & $0.852[0.604 ; 0.99]$ & $0.533[0.16 ; 0.895]$ & $0.202[0.119 ; 0.894]$ \\
\hline$S p \mathrm{HI}$ & $0.961[0.951 ; 0.974]$ & $0.961[0.961 ; 0.973]$ & $0.962[0.948 ; 0.979]$ & $0.961[0.951 ; 0.974]$ & $0.962[0.951 ; 0.974]$ & $0.962[0.947,0.98]$ & $0.959[0.937 ; 0.978]$ \\
\hline pi Ada'a, wet & $0.063[0.041 ; 0.097]$ & $0.0620[0.041 ; 0.095]$ & $0.100[0.046 ; 0.267]$ & $0.063[0.041 ; 0.097]$ & $0.06[0.039 ; 0.094]$ & $0.1[0.048 ; 0.398]$ & $0.278[0.055 ; 0.613]$ \\
\hline pi Ada'a, dry & $0.132[0.097 ; 0.191]$ & $0.132[0.097 ; 0.190]$ & $0.210[0.105 ; 0.52]$ & 0.132 [0.098; 0.191] & $0.127[0.093 ; 0.185]$ & $0.211[0.111 ; 0.79]$ & $0.578[0.121 ; 0.978]$ \\
\hline pi ATJK, wet & $0.043[0.025 ; 0.072]$ & $0.042[0.025 ; 0.071]$ & $0.069[0.029 ; 0.187]$ & $0.043[0.025 ; 0.072]$ & $0.04[0.023 ; 0.069]$ & $0.07[0.03 ; 0.274]$ & $0.18[0.036 ; 0.422]$ \\
\hline pi ATJK, dry & $0.062[0.038 ; 0.099]$ & $0.061[0.038 ; 0.098]$ & $0.096[0.042 ; 0.255]$ & $0.062[0.038 ; 0.1]$ & $0.058[0.035 ; 0.097]$ & $0.097[0.045 ; 0.373]$ & $0.259[0.051 ; 0.594]$ \\
\hline rhoD & $0.461[-0.055 ; 0.894]$ & $0.467[0.052 ; 0.894]$ & $0.763[0.016 ; 0.949]$ & $0.442[-0.062 ; 0.886]$ & $0.382[-0.061 ; 0.873]$ & $0.762[0.063 ; 0.944]$ & $0.785[0.241 ; 0.935]$ \\
\hline
\end{tabular}

Bold figures highlight posterior distribution that differ substantially from those obtained with informative priors for all parameters 
Table 6 Sensitivity analysis: posterior estimates for the sensitivity and specificity of bELISA and HI tests, and prevalence, in an eight populations Bayesian model including conditional dependence between test results for truly positive animals.

\begin{tabular}{|c|c|c|c|}
\hline \multirow[t]{2}{*}{ Parameter } & $\begin{array}{c}\text { Informative priors(as } \\
\text { in Table 1) }\end{array}$ & $\begin{array}{c}\text { Non informative } \\
\text { priors for } \\
\text { prevalences } \\
\text { (poor convergence) }\end{array}$ & $\begin{array}{l}\text { Non informative priors for } \\
\text { test performance } \\
\text { parameters (poor } \\
\text { convergence) }\end{array}$ \\
\hline & median $[95 \% \mathrm{PCI}]$ & median $[95 \% \mathrm{PCI}]$ & median $[95 \% \mathrm{PCI}]$ \\
\hline Se bELISA & $0.803[0.453 ; 0.983]$ & $0.489[0.095 ; 0.974]$ & $0.185[0.122 ; 0.341]$ \\
\hline$S p$ bELISA & $0.983[0.972 ; 0.995]$ & 0.98 [0.959; 0.993] & $0.977[0.962 ; 0.99]$ \\
\hline $\mathrm{Se} \mathrm{Hi}$ & $0.729[0.419 ; 0.913]$ & $0.453[0.078 ; 0.9]$ & $0.167[0.105 ; 0.316]$ \\
\hline$S p \mathrm{HI}$ & $0.96[0.95 ; 0.971]$ & $0.951[0.854 ; 0.968]$ & $0.943[0.917 ; 0.959]$ \\
\hline pi Ada'a, wet market & $0.091[0.036 ; 0.195]$ & $0.174[0.039 ; 0.784]$ & $0.262[0.1 ; 0.511]$ \\
\hline pi Ada'a, wet household & $0.067[0.041 ; 0.125]$ & $0.114[0.043 ; 0.841]$ & $0.298[0.138 ; 0.543]$ \\
\hline pi Ada'a, dry market & $0.081[0.032 ; 0.181]$ & $0.145[0.034 ; 0.555]$ & $0.205[0.08 ; 0.403]$ \\
\hline pi Ada'a, dry household & $0.159[0.111 ; 0.288]$ & $0.27[0.116 ; 0.968]$ & $0.622[0.347 ; 0.817]$ \\
\hline pi ATJK, wet market & $0.043[0.011 ; 0.117]$ & $0.075[0.01,0.499]$ & $0.115[0.026 ; 0.298]$ \\
\hline pi ATJK, wet household & $0.049[0.028 ; 0.095]$ & $0.086[0.029 ; 0.676]$ & $0.199[0.092 ; 0.378]$ \\
\hline pi ATJK, dry market & $0.043[0.01 ; 0.126]$ & $0.061[0.009 ; 0.318]$ & $0.087[0.019 ; 0.229]$ \\
\hline pi ATJK, dry household & $0.077[0.046 ; 0.146]$ & $0.134[0.048 ; 0.996]$ & $0.387[0.163 ; 0.755]$ \\
\hline rhoD & $0.664[0.002 ; 0.947]$ & $0.826[0.075 ; 0.933]$ & $0.9[0.812 ; 0.974]$ \\
\hline
\end{tabular}

Bold figures highlight posterior distribution that differ substantially from those obtained with informative priors for all parameters

\section{Discussion}

This study evaluated the performance of three serological tests (bELISA, iELISA and HI) for the detection of NDV antibodies in unvaccinated village chickens under field conditions, in the absence of 'gold standard' test, using a Bayesian framework. A modification of the two population approach was used, because sampling was done both in markets and households during two different seasons and in two distinct Woredas, which could potentially produce 
variation in seroprevalence according to these factors. A set of candidate models differing with regard to prevalence variation pattern were thus fitted to the serological data. Test performance parameters were assumed to be similar across woredas, surveys (household or market) and seasons (wet and dry). Disease status is ideally judged by the presence or absence of the disease causing pathogen, but since we used serological tests, the prevalence estimations produced should be interpreted in terms of frequency of presence of antibodies which reflects NDV infection incidence or exposure over a time period. However, as the period of persistence of NDV antibodies in chickens has not been clearly established, it would be difficult to determine whether seropositive individuals were actually infected at the time of sampling or the stage of the disease in individuals that were indeed infected. In other word, it is reasonable to consider that serological prevalence reflects the frequency of infections over a time period prior to sampling. However, the limits of this time period are difficult to determine.

The analyses revealed that bELISA performed better than the HI test with respect to its $S e$ and $S p$ (Table 3). The $S e$ of bELISA and HI were comparable to those reported during validation (Czifra et al., 1996). As for iELISA, the extremely high proportion of samples that tested positive for this test as compared to bElisa or HI suggested that in the specific context of the present study, iELISA dramatically lacked specificity. The validity of such a conclusion however relies on the reproducibility of the tests outcomes. The samples have initially been tested at National Animal Health Diagnostic and Investigation Center (NAHDIC). At this stage a high proportion of positive results were obtained for iELISA but not for bELISA or HI. Since the result of iELISA triggered doubt and it was decided to send a certain number of sample (100) as well as the same iELISA and bELISA kits to the poultry reference center of University of Pretoria for cross checking. The result for iELISA was again appeared quite different than those of bELISA and HI, indicating very low specificity of 
iELISA (high number of positive reaction). The $S e$ and $S p$ of a serological test are affected by several factors, including the conditions under which the test is performed, the stage or duration of the infection, the presence of cross reactions from organisms of similar antigenic structure and cut-off values used to interpret tests (Greiner and Gardner, 2000). Whether the testing is performed on naturally infected or experimentally infected animal may also play a role, since it has been established that, for avian influenza, HI performs more accurately in naturally infected than in experimentally infected birds (Comin et al., 2012). The sensitivity of HI is affected by the cut-off values used $\left(4 \log _{2}\right)$, but lower cut-off values are considered prone to non-specific reactions (OIE, 2008b). A higher cut-off value $\left(>4 \log _{2}\right)$ than this, would increase the $S p$ of results but at the expense of decreased $S e$. Moreover, the $S e$ of the HI test might be more dependent on the antigenic characteristics of virus strains, whereas the $S e$ of the bELISA is less variable in response to strain variation (Czifra et al., 1996). The respective methods of the $\mathrm{HI}$ and the ELISA tests imply that antibodies to different antigenic determinants of the virus are measured in each assay. While the former test specifically detects antibodies directed against epitopes of the haemagglutinin neuraminidase protein of APMV-1, the ELISA detects mostly a wider spectrum of antibodies specific for different viral proteins (Czifra et al., 1998), unless designed to detect specific antibodies against a selected structural protein. Furthermore, it has been observed, in avian influenza diagnosis, that the performance of HI test is strongly influenced by the homology or relatedness between the reference viral antigens and the virus isolate or antibodies to be tested (Escorcia et al., 2010) and this may be equally valid for NDV. Blocking antibodies developed against the PMV-1 epitope, like the one used in the bELISA, are specific for the NDV infection in any host and at any age (Koch et al., 1998). The onset and amount of antibody production against the different proteins of NDV might vary with the time after infection and affect test parameters and hence estimation of prevalence. A time dependent discrepancy between the 
HI test and ELISA has been observed for the detection of antibodies to a haemagglutinating NDV antibody in domestic geese and Muscovy ducks after immunization (Hauslaigner et al., 2009). It was suggested that this phenomenon was due to different test methods: both IgG and $\operatorname{IgM}$, which predominates during the first 2 weeks of a primary immune response, are able to agglutinate viral particles (Davison et al., 2008) and are therefore likely to be measured by the HI test. Furthermore, Hauslaigner et al. (2009) suggested that the differences in the antibody pattern leads to a greater number of false-negative results in the HI test than in the ELISA, more specifically at later time points after virus contact, and that the ELISA provides a higher detection level than the HI test, which is advantageous in surveillance programmes. The results of our study support this. Since HI is commonly used in NDV antibody diagnosis its performance has rarely been questioned. However, studies have shown that the variation between the quantitative test results of different laboratories using the NDV HI test was higher (about double) compared with the variation within commercial ELISA systems (de Wit et al., 2007).

There was no published information available on $S e$ and $S p$ of the iELISA and hence the iELISA priors were based on the manufacturer's claim of $S e$ and $S p>95 \%$ (Laura Fournier, 2011, personal communication). Considering the high proportion of positive test results for iELISA, although not evaluated in models, it was concluded that iELISA had a very low specificity in the context of the present study. There could be several reasons for this. The apparently much lower specificity of iELISA on field samples might be attributed to use of inappropriate low cut-off values that categorized most field samples as positive. It may be that a different cut-off should be used in village chickens. The presence of other crossreacting APMV in the field, although not investigated, is another possible explanation for the low $S p$ of the iELISA since other APMVs were observed to increase ELISA absorbance (Adair et al., 1989). Cross reactions between APMV-1 and other APMV subtypes, including 
APMV-2, APMV-3, and APMV-7, have been demonstrated in the HI test and in an ELISA involving whole virus antigen (Alexander et al., 1983; Nayak et al., 2012). There might also be a background reaction that has increased the OD values of the sample in iELISA assay. It is, therefore, plausible that a similar phenomenon might be exhibited using the iELISA, unlike the bELISA, where specific monoclonal antibodies are used. The apparently poor $S p$ of iELISA in this study calls into question its suitability for field investigation of village chicken NDV antibody status or for surveillance in unvaccinated flocks in Ethiopia.

Two test outcomes for a given animal are likely to be correlated if both tests measure a similar biological phenomenon (Gardner et al., 2000). Since both ELISA and HI tests are based on the same principle, namely the detection of humoral immune responses induced by NDV infection, although different antigenic proteins are targeted a certain degree of dependence between test results was expected. Indeed, the results of Bayesian modelling reported here suggest that the results of bELISA and HI tests are positively correlated for truly positive animals. However, wide posterior distribution for the correlation parameter indicates large uncertainty regarding the strength of the dependence.

Model selection suggested that serological prevalence varied between seasons and woredas. The prevalence estimates of NDV antibody varied from $4.3 \%$ (95\% PCI: $2.5,7.2 \%$ ) in the ATJK woreda during the wet season to $13.2 \%$ (95\% PCI: 9.7, 19.1\%) in the Ada'a woreda during the dry season. Previously, Getachew (2009) using the same bELISA reported a comparable seroprevalence of $5.6 \%$ (95\% CI: 3.2, 8.8\%), in village chickens in south west Shewa, Ethiopia. Considering the village chickens were not vaccinated, the seropositive results obtained at villages were likely to be a true reflection of field NDV seroconversion status of individual chickens. The apparent low prevalence is likely the reflection of the fact that village chickens in the study area were more affected by virulent virus with likely high 
mortality and few seropositive survivors. The estimate of $S e$ and $S p$ for the tests (bELISA and HI) obtained in the present study is considered to allow unbiased estimates of the prevalence in the study area. The prevalence estimates are consistent with reports from other African countries, i.e. a seasonal variation in incidence of ND, which was higher during the dry season (Sa'idu et al., 2006; Nwanta et al., 2006; Otim et al., 2007; Miguel et al., 2013).

In fact, for the assumption of differing prevalence, as in the latent class analysis, a bigger difference was considered better for the analysis (Toft et al., 2005). But in the present study since the prevalence are not very different, this might have undermined the assumption of differing prevalence and could be regarded as a limitation. A potential source of bias was the possibility that some individual chickens may have been re-tested during the second sampling. Moreover, the tests were applied on unvaccinated chickens presumably at different stages of infection. Both ELISA and HI tests are subject to false negative results that may result from low antibody titre, because of the decline in antibody titre after natural infection associated with phase of infection (Bell and Mouloudi, 1988). Schelling et al. (1999), applying bELISA in Swiss backyard flocks, observed poor reproducibility and low $S p$ of the test in a very low disease prevalence situation with weakly positive samples while Gohm et al. (1999), checking sera from the specific pathogen free and immunized chickens, found an excellent $S e$ and $S p$ for bELISA.

According to sensitivity analysis, it appeared that the selected model generated consistent parameter estimates and was not very sensitive to selection of priors (Table 5). The only requirement for obtaining consistent posterior distribution was the use of a moderately informative prior distribution for the sensitivity of the bELISA test. The limitation in this respect is mainly associated with the paucity and/or accuracy of the available information which has been used as prior. However, from a purely Bayesian perspective, one is simply 
modeling uncertainty about parameters and does not expect ultimately to know the precise values unless the quality of the prior information is extremely precise (Enue et al., 2000). Nevertheless, the difficulty of evaluating models that considers dependence between tests, without sufficiently informative priors has been noted (Toft et al., 2005). Although it was not validated on extensive data sets, the approach followed in this study, combining data from different sources (market and household surveys) in one model, could produce valid estimates of test parameters across different populations. Further evaluation using data from more distant geographical areas, preferably with differing prevalence, would contribute to better diagnostic test parameter estimates since it has been shown that estimates for diagnostic $S e$ and $S p$ may vary among populations and/or subpopulations of animals, conditional on the distribution of influential covariates or even the sampling strategy (Greiner and Gardner, 2000).

\section{Conclusion}

Serological tests are a useful tool to assess NDV infection status of a population, indirectly based on past exposure, particularly where other diagnostic techniques such as RT-PCR are not readily available. Our estimates of $S e$ and $S p$ of both bELISA and HI show that they can be used for diagnosis and effective surveillance of NDV antibodies in unvaccinated chickens under field conditions similar to those in our study. Given ease of automation, bELISA may be preferred. On the other hand, given its high specificity, HI may be used as a confirmatory test if an ELISA of low $S p$ like iELISA is used for screening. This is especially helpful to assess situations where individual chicken NDV antibody is generally considered low, following serial test interpretations to improve the specificity and the positive predictive values. The use of local virus as antigen and locally-produced antiserum in HI should be investigated. The presence of other potentially cross-reacting APMVs in village chickens also 
requires further investigation. To avoid biased results or erroneous inferences, serological tests should ideally first be evaluated in a population similar to the one in which they will be used.

\section{Conflict of interest}

No conflict of interest to be declared

\section{Acknowledgments}

This study was funded by the French Ministry of Foreign Affairs (MAE) via the FSP project [GRIPAVI 2006-26]. Authors would like to thank staff members at NAHDIC for assistance during fieldwork.

\section{References}

Adair B.M., McNulty, M.S., Todd, D., Connor, T.J., Burns, K., 1989. Quantitative estimation of Newcastle disease virus antibody levels in chickens and Turkeys by Elisa. Avian Pathol. $18,175-192$

Ahmed, F., 2010. An application of a Bayesian approach in diagnostic testing problems in the absence of a gold standard. Drug Info. J. 44, 453-462.

Alexander, D.J., 1998. Newcastle disease and other avian paramyxoviruses. In: A Laboratory Manual for the Isolation and Identification of Avian Pathogens 4th edition. Swayne, D.E. Glisson, J.R. Jackwood, M.W. Pearson J.E., Reed, W.M.(Eds). American Association of Avian Pathologists, Kennet Square, USA. pp 156-163.

Alexander, D.J., Bell, J.G., Alders, R.G., 2004. A technology review, Newcastle disease. FAO Animal production and health book, No. 161, FAO, Rome. 
Alexander, D.J., Pattison, M., Macpherson, I., 1983. Avian paramyxoviruses of PMV-3 serotype in British turkeys. Avian Pathol. 12, 469-482.

Beard, C.W., Wilkes, W.J., 1985. A comparison of Newcastle disease HI test results from diagnostic laboratories in the South Eastern United States. Avian Dis. 29, 1048-1056.

Bell, J.G., Mouloudi, S., 1988. A reservoir of virulent Newcastle disease virus in village chicken flocks. Prev. Vet. Med. 6, 37-42.

Branscum, A.J., Gardner, I.A., Johnson, W.O., 2005. Estimation of diagnostic-test sensitivity and specificity through Bayesian modelling. Prev. Vet. Med. 68, 145-163

Cadman, H.F., Kelly P.J., de Angelis N.D., Rohde, C., Collins, N., Zulu, T., 1997. Comparison of enzyme-linked immunosorbent assay and haemagglutination inhibition test for the detection of antibodies against Newcastle disease virus in ostriches (Struthio camelus). Avian Pathol. 26, 357-363

Cattoli, G., Monne. I., 2009. Molecular Diagnosis of Newcastle Disease Virus. In: Ilaria Capua, I., Alexander, D.J (Eds), Avian Influenza and Newcastle disease. A field and laboratory manual, Springer, Verlag Italia, PP 127-132.

Comin, A., Toft, N., Stegeman, A., Klinkenberg, D., Marangon S., 2013. Serological diagnosis of avian influenza in poultry: is the haemagglutination inhibition test really the 'gold standard'? Influenza Other Respir. Viruses, 7(3), 257-64.

Chaka, H., Goutard, F., Bisschop, S.P.R., Thompson, P.N., 2012. Seroprevalence of Newcastle disease and other infectious diseases in backyard chickens at markets in Eastern Shewa Zone, Ethiopia. Poult. Sci., 91: 862-869.

Chaka' H., Goutard, F, Gil, P., Abolnik, C., Servan de Almeida, R., Bisschop, S.P.R., Thompson, P. N., 2013. Serological and molecular investigation of Newcastle disease in 
household chicken flocks and associated markets in Eastern Shewa zone, Ethiopia. Trop. Anim. Health Prod. 45, 5-14.

Czifra, G., Nilsson, M., Alexander, D.J., Manvell, R., Kecskeméti, S., Engström, B.E., 1996. Detection of PMV-1 specific antibodies with a monoclonal antibody blocking enzyme-linked immunosorbent assay. Avian Pathol. 25, 691-703.

Czifra G., Mészáros J., Horváth E., Moving, V., Engström, B.E., 1998. Detection of NDVspecific antibodies and the level of protection provided by a single vaccination in young chickens. Avian Pathol. 27, 562-565.

Davison, F., Katharine, E., Magor, K., Kaspers, B., 2008. Structure and evolution of Avian immunoglobulins. In: Davison, F., Kaspers, B. Schat, K.A. (Eds): Avian Immunology, Elsevier Ltd, Amsterdam, PP 107-127.

de Wit, J.J., van de Sande, H. W., Counotte, G.M., Wellenberg, G.J., 2007. Analyses of the results of different test systems in the 2005 global proficiency testing schemes for infectious bursal disease virus and Newcastle disease virus antibody detection in chicken serum. Avian Pathol. 36, 177-83.

Enùe, C., Georgiadis, M.P., Johnson, W.O., 2000. Estimation of sensitivity and specificity of diagnostic tests and disease prevalence when the true disease state is unknown. Prev. Vet. Med. 45, 61-81.

Escorcia, M, Carrillo-Sa’n nchez, K., March-Mifsut, S., Chapa, J., Lucio, E., Nava, G., 2010. Impact of antigenic and genetic drift on the serologic surveillance of H5N2 avian influenza viruses. BMC, Vet. Res. 6:57.

Gardner, I.A., Stryhn, H., Lind, P., Collins, M.T., 2000. Conditional dependence between tests affects the diagnosis and surveillance of animal diseases. Prev. Vet. Med. 45, 107-122. 
Getachew, B., 2009. Isolation, identification and seroprevalence of Newcastle disease virus in village chicken in South West Shewa, Ethiopia, (unpublished Msc Thesis, Addis Ababauniversity, Ethiopia).

Gohm, D.S., Barbara Thür, B., Audige, L., Hofmann, M.A., 1999. A survey of Newcastle disease in Swiss laying-hen using serological testing and simulation modelling. Prev. Vet. Med. 38, 277-288.

Gohm, D.S., Barbara Thür, B., Hofmann, M.A., 2000. Detection of Newcastle disease virus in organs and faeces of experimentally infected chickens using RT-PCR. Avian Pathol. 29, $143-152$.

Greiner, M., Gardner, I.A., 2000. Application of diagnostic tests in veterinary epidemiologic studies. Prev. Vet. Med. 45, 43-59.

Häuslaigner, R., Sonnenburg, J., Kothlow, S., Kaspers, B., Staubach, C., Grund, C., 2009. Evaluation of an indirect enzyme-linked immunosorbent assay to study the specific humoral immune response of Muscovy ducks (Cairina moschata) and domestic geese (Anser anser var. domestica) after vaccination against Newcastle disease virus, Avian Pathol. 38, 89-95.

Miguel, E., Grosbois, V., Berthouly-Salazar, C., Caron, A., Cappelle, J., Roger, F., 2013. A meta-analysis of observational epidemiological studies of Newcastle disease in African agrosystems, 1980-2009. Epidemiol. Infect. 141, 1117-1133

Nayak, M., Militino Dias, F., Kumar, S., Paldurai A., Collins P.L., Samal, S.K., 2012., Avian paramyxovirus serotypes 2-9 (APMV-2-9) vary in the ability to induce protective immunity in chickens against challenge with virulent Newcastle disease virus (APMV-1). Vaccine. 30, $2220-2227$ 
Lipkind, M., Shihmanter, E., 1986. Antigenic relationships between avian paramyxoviruses 1. Quantitative characteristics based on haemagglutination and neuraminidase inhibition tests. Archi. Virol. 89, 89-11

OIE. 2008. Newcastle disease. In: Manual of diagnostic test and vaccine for Terrestrial animals. $6^{\text {th }}$ ed., Volume 1 and2. Pp576- http://www.oie.int/international-standardsetting/terrestrial-manual/ (access-on July 2010).

Koch, G., Czifra, G., Engstrom, B. E., 1998. Detection of Newcastle disease virus-specific antibodies in ostrich sera by three serological methods. Vet. Rec. 143, 10-12.

Regasa, C., Fufa, D. Berihanu, S., Hunduma, D., 2007. Sero-prevalence of Newcastle disease in backyard chickens in mid rift valley of Oromia, Ethiopia. Work Poster. In: Camus, E., Cardinale, E., Dalibard, C., Martinez D., Renard, J.F, Roger F. (Eds.). Proceedings of the 12th International conference of the AITVM, Montpellier, France, 20-22 August, 2007.

Schelling, E., Thur, B., Griot, C., Audige, L., 1999. Epidemiological study of Newcastle disease in backyard poultry and wild bird populations in Switzerland. Avian Pathol. 28, 263272.

Spiegelhalter, D., Thomas, A., Best, N., Lunn, D., 2003. WinBUGS User Manual Version 1.4. January 2003. http://www.mrc-bsu.cam.ac.uk/bugs.

Suess, E.A., Johnson, W.O., Gardner, I.A., 2002. Hierarchical Bayesian model for prevalence inferences and determination of a country's status for an animal pathogen. Prev. Vet. Med. $55,155-171$.

Suess, E.A., Johnson, W.O., Gardner, I.A., 2002. Hierarchical Bayesian model for prevalence inferences and determination of a country's status for an animal pathogen. Prev. Vet. Med. $55,155-171$. 
Tabidi M.H., Makkawi, A. Mahasin, E., Ali, A.S., 2004. Comparative Evaluation of Haemagglutination Inhibition Test and Enzyme-linked Immunosorbent Assay for detection of antibodies against Newcastle disease vaccine in Broiler chicks. Int. J. Poult. Sci. 3, 668-670. Terregino, C., Capua, I. 2009. Conventional Diagnosis of Newcastle Disease Virus Infection. In: Capua I., Alexsande D.J (Eds). Avian Influenza and Newcastle disease, A field and laboratory manual. Springer, PP 123-126.

Toft, N, Jørgensen, E, Gettinby, G, Højsgaard, S., 2005. Diagnosing diagnostic tests: evaluating the assumptions underlying the estimation of sensitivity and specificity in the absence of a gold standard. Prev. Vet. Med. 68, 9-33.

Toft, N, Innocent, G.T., Gettinby, G., Reid, S.W.J., 2007. Assessing the convergence of Markov Chain Monte Carlo methods: an example from evaluation of diagnostic tests in absence of a gold standard. Prev. Vet. Med. 79, 244-256.

Zeleke, A., Sori, T., Gelaye, E., Ayelet, G., 2005. Newcastle disease in Village Chickens in the southern and Rift valley Districts in Ethiopia. Int. J. Poult. Sci. 4, 507-510. 


\section{Appendix A. Supplementary data}

\section{Blocking ELISA}

Blocking ELISA was performed using Svanovir ${ }^{\mathrm{TM}}$ NDV-Ab test kits (Svanova Biotech, Uppsala, Sweden) using the procedure described by manufacturer and validated by Czifra et al. (1996). The test was developed to detect specific serum antibodies against a well-conserved epitope present in APMV-1 strains. Briefly, NDV-coated ELISA plates were first incubated with the samples, allowing NDV specific antibodies present in the sample to bind to epitopes. If NDV specific antibodies were absent, the epitopes remained free. After washing, horseradish peroxidase (HRP) conjugate monoclonal antibody $(\mathrm{mAb})$ was added to the wells and the binding of the conjugate $\mathrm{mAb}$ was visualized with a substrate solution. A negative result was indicated by a strong colour change. The sample and control optical density (OD) values were read using an ELISA reader at $450 \mathrm{~nm}$. The antibody titres were calculated as percentage inhibition (PI) for the positive control as well as the samples, using the formula

$\mathrm{PI}=\left[\left(\mathrm{OD}_{\text {Negative control }}-\mathrm{OD}_{\text {Sample }}\right) / \mathrm{OD}_{\text {Positive control }}\right] \times 100 / \mathrm{OD}_{\text {Negative Control }}$

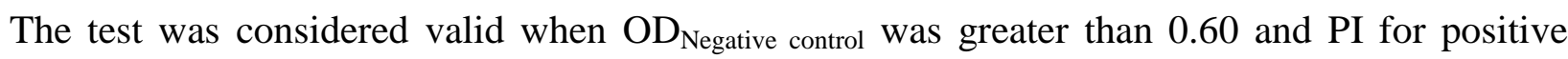
control solution was greater than 40 . All sera that blocked the binding of the specific monoclonal antibody with a $40 \%$ inhibition (PI $>40$ ) were considered bELISA positive.

\section{Indirect ELISA}

The LSIVET AVI NDV ${ }^{\circledR}$ ELISA was obtained from Laboratoire Service International (LSI, Lissieu, France) and is based on the principle of indirect ELISA (iELISA) and designed to be used on individual poultry serum. The iELISA works on the principle of recognition of antiNDV antibodies, attached to a plate coated with viral antigen (nucleoprotein), by antibodies 
produced in another species against chicken antibodies. Briefly, samples were added to the NDV antigen coated plate. If present, specific antibodies against NDV bound to antigen forming antigen-antibody complexes. After washing anti-chicken IgY/HRP-labeled conjugate was added, binding to any chicken antibodies attached to the plate. Unbound conjugate was removed by washing, after which a chromogen substrate was added. This anti-chicken antibody is conjugated to an enzyme (peroxydase) that catalyses a reaction, causing a change of colour which was read quantitatively on a photospectrometer at $405 \mathrm{~nm}$.

For each tests the sample-to-positive (S/P) ratios were calculated from absorbance values by the formula:

$$
\mathrm{S} / \mathrm{P} \text { ratio }=\left[\mathrm{OD}_{\text {Sample }}-\mathrm{OD}_{\text {Negative control mean }}\right] /[\mathrm{OD} \text { Positive control mean }-\mathrm{OD} \text { Negative control mean }]
$$

The test was considered valid if $\mathrm{OD}_{\text {Positive control mean }}>0.5$ and $\left(\mathrm{OD}_{\text {Positive control mean }} / \mathrm{OD}_{\text {Negative control }}\right.$ mean) > 4. All samples with $\mathrm{S} / \mathrm{P}$ ratio $\geq 0.234$ were considered positive (manufacturer's recommendation).

Haemagglutination inhibition test

The HI test is based on the principle that the haemagglutinin on the viral envelope can bring about the agglutination of chicken red blood cells (RBC) and that this can be inhibited by specific antibodies (Alexander et al., 2004). The test was performed as described by the World organization for Animal Health (OIE, 2008). The antigen used was a commercial NDV LaSota strain provided by LSI, France and reconstituted in sterile distilled water, while positive and negative control NDV strain La Sota antisera were procured from GD Animal Health Service Ltd, Deventer, The Netherlands. Antigen was diluted to contain 4 haemagglutination units 
(HAU). The test was performed on V-bottom microtiter plates. The presence or absence of agglutination was accurately assessed by tilting the plates. Only those wells in which the RBCs streamed at the same rate as the control wells (containing RBCs and PBS only) were considered to show inhibition. The titres were expressed as $\log _{2}$ of the reciprocal of the highest dilution of serum giving complete inhibition of 4 HAU. Sera with HI antibody $\geq 4 \log _{2}$ were considered positive for NDV antibody (OIE, 2008b). 